\title{
Actualización \\ Manejo de la bronquiolitis en niños
}

\author{
(Síntesis del Informe de Evaluación de Tecnología: "Management of Bronchiolitis in Infants and Children" \\ Agency for Healthcare Research and Quality ${ }^{1}$ )
}

\section{INTRODUCCIÓN Y EPIDEMIOLOGÍA}

En Argentina, las infecciones respiratorias agudas (IRA) constituyen uno de los principales problemas de salud de los niños menores de cinco años, ocasionando casi el $50 \%$ de las internaciones y de las consultas ambulatorias, y siendo la primera causa de muerte en menores de un año (104/100 000 nacidos vivos en 1995). Cada año más del $20 \%$ de los niños desarrollan infección respiratoria aguda baja (IRAB) y cerca del $3 \%$ de los menores de un año se internan por bronquiolitis, su forma más común.

La bronquiolitis se caracteriza por una inflamación difusa y aguda de las vías aéreas inferiores definida como un primer episodio de sibilancias con evidencia clínica de infección viral en menores de dos años. El virus sincicial respiratorio (VSR) es el principal responsable $(70 \%$ global y $90 \%$ en invierno) aunque también la ocasionan el adenovirus y el parainfluenza, aún en época no invernal.

Los factores de riesgo para desarrollar la enfermedad son: edad menor a seis meses, falta de lactancia materna, hacinamiento, presencia de hermanos mayores o convivientes fumadores y concurrencia a guardería.

Son predictores de mal pronóstico e internación prolongada: edad menor a tres meses, desnutrición severa y comorbilidades (cardiopatía congénita, enfermedad pulmonar o neuromuscular crónica, inmunodeficencia); estos grupos tienen tres veces más riesgo de padecer una bronquiolitis grave.Un 10 a 15\% de las bronquiolitis requiere internación (menos del $3 \%$ en niños previamente sanos, $25 \%$ en prematuros y hasta $50 \%$ en portadores de displasia broncopulmonar). La mortalidad global es menor al $1 \%$ pero puede llegar hasta $37 \%$ en cardiópatas.

\section{FISIOPATOGENIA Y PRESENTACIÓN CLÍNICA}

La inflamación aguda, edema y necrosis de la mucosa respiratoria, el aumento de la producción de moco y el espasmo bronquial, generan obstrucción de la pequeña vía aérea, su característica más saliente. Puede haber rinorrea, fiebre, taquipnea, tos, rales subcrepitantes, sibilancias y uso de músculos accesorios de la respiración con tiraje y aleteo nasal.

\section{REVISIÓN SISTEMÁTICA DE LA AHRQ}

La alta prevalencia de la bronquiolitis y su severidad en grupos vulnerables generan un alto costo y un impacto considerable en la salud pública. La evidencia sobre la efectividad de las distintas intervenciones es conflictiva y muchas conductas habituales carecen de eficacia comprobada.La American Academy of Pediatrics y la American Academy of Family Physicians solicitaron a la Agency for Healthcare Research and Quality la realización de esta revisión sistemática sobre el manejo de la bronquiolitis, que fue desarrollada por el Evidence-based Practice Center de la University of North Carolina.

\section{PREGUNTAS CLAVE PARA LA REVISIÓN SISTEMÁTICA}

1. ¿Cuál es la efectividad de las herramientas diagnósticas (radiología de tórax y tests de laboratorio) en la bronquiolitis?

2. ¿Cuál es la efectividad de la farmacoterapia? ¿Algún agente es superior a los demás?

3. ¿Cuál es el rol de la profilaxis en la prevención de la bronquiolitis en niños? ¿Qué subpoblaciones se beneficiarían con la profilaxis?

4. ¿Cuál es la costo-efectividad de la terapéutica profiláctica para la bronquiolitis en prematuros o niños con comorbilidades?

\section{Metodología de la revisión sistemática}

Se localizaron todos los estudios originales publicados de 1966 a 2002 en idioma inglés, indexados en MEDLINE, Cochrane y Health Economic Evaluation Database, que respondieran a las preguntas formuladas. Para tratamiento y profilaxis la revisión se limitó a los ensayos clínicos aleatorizados (ECA) mientras que para diagnóstico se analizaron también otros estudios prospectivos; la costoefectividad se valoró mediante estudios de evaluación económica. Se excluyeron las series pequeñas $(n<10)$. Se identificaron 744 resúmenes; el análisis de los trabajos completos seleccionó 83 que fueron incluidos en la revisión (16 para diagnóstico, 52 para tratamiento, 9 para profilaxis y 6 para costo-efectividad).

\section{Efectividad de las herramientas diagnósticas para bron-} quiolitis

La bronquiolitis es una enfermedad de diagnóstico clínico basado en los antecedentes y el examen físico y bien definida por criterios aceptados como los de Engle-News ${ }^{2}$, Court ${ }^{3}$ o Denny-Clyde ${ }^{4}$. No existe un test de referencia que confirme la sospecha clínica. La severidad se relaciona con el grado de hipoxemia y el cuadro puede clasificarse como leve (manejo ambulatorio) moderado (requiriendo internación) o severo (insuficiencia respiratoria y necesidad de asistencia respiratoria mecánica).Los revisores no encontraron literatura específica sobre el diagnóstico de bronquiolitis; solamente se sintetizó la evidencia sobre el rol de marcadores de severidad y exámenes complementarios radiológicos, bioquímicos y virológicos involucrados.

\section{Predictores de severidad o mal pronóstico}

Existen numerosas escalas clínicas que miden la severidad del cuadro, utilizando parámetros como frecuencia respiratoria y cardíaca (FR y FC), esfuerzo respiratorio, intensidad de las sibilancias $u$ oxigenación; sin embargo, no todas han sido validadas. En nuestro país, la más utilizada es el score de Tal ${ }^{5}$ que valora la FR y FC, sibilancias y tiraje para asignar grados de severidad. Otros puntajes clínicos de severidad son el Respiratory Assessment Change Score (RACS) el Respiratory Distress Assessment Instrument (RDAI), el Respiratory Distress Score (RDS) y el Lower Respiratory Tract Illness/Infection Score (LRI).Los principales resultados de estudios sobre indicadores de severidad se muestran en la TABLA 1. 
Tabla 1: estudios sobre predictores de severidad.

\begin{tabular}{|c|c|c|c|}
\hline AUTOR & INDICADORES EXAMINADOS & RESULTADOS PREDICHOS & PREDICTORES SIGNIFICATIVOS \\
\hline Cherian et al., 19976 & $\begin{array}{l}\text { 1. } F R(\geq 60 / \text { min en }<2 \text { meses de edad, } \geq 50 / \mathrm{min} \\
\text { de } 2-12 \text { meses, } \geq 40 \text { en }>12 \text { meses }) \text {. } \\
\text { 2. Presencia de tiraje subcostal }\end{array}$ & $\begin{array}{l}\text { IRAB en niños desnutridos (por anomalías } \\
\text { radiológicas o auscultatorias). }\end{array}$ & $\begin{array}{l}\text { La sensibilidad y especificidad de la taquipneay/o tira } \\
\text { je para detectar IRAB no varió según el estado nutri- } \\
\text { cional. }\end{array}$ \\
\hline Dawson et al., 19907 & $\begin{array}{l}\text { Hallazgos radiológicos (hiperinsuflación, atelectasias } \\
\text { e infiltrados). }\end{array}$ & $\begin{array}{l}\text { Puntaje clínico (leve, moder ado, severo o muy } \\
\text { severo). }\end{array}$ & $\begin{array}{l}\text { Sin correlación entre hallazgos radiológicos y severi- } \\
\text { dad. }\end{array}$ \\
\hline Mulholland et al., 19908 & $\begin{array}{l}\text { 1. Datos demográficos } \\
\text { 2. Cianosis } \\
\text { 3. Rales subcrepitantes } \\
\text { 4. Tiraje } \\
\text { 5. } \mathrm{RR}>50 / \mathrm{min} \\
\text { 6. } \mathrm{FC}>150 / \mathrm{min} \\
\text { 7. } \mathrm{Hígado}>2 \mathrm{~cm} \text { por debajo del reborde costal } \\
\text { 8. } \mathrm{SaO}_{2}<90 \% \\
\text { 9. } \mathrm{PaO}_{2}<60 \mathrm{mmHg} \\
\text { 10. } \mathrm{PaCO}>45 \mathrm{mmHg} \\
\text { 11. Status VSR. }\end{array}$ & $\begin{array}{l}\text { Severidad al ingreso valorada por oximetría y } \\
\text { gases arteriales } \\
\text { Requerimiento de } \mathrm{O}_{2} \text { durante la admisión. }\end{array}$ & $\begin{array}{l}\text { Indicadores de severidad al ingreso: } \\
\text { 1. Corta edad } \\
\text { 2. đanosis } \\
\text { 3. Estridores subcrepitantes } \\
\text { Predictores de ingreso para O2terapia: } \\
\text { 1. Corta edad } \\
\text { 2. Canosis } \\
\text { 3. Estridores subcrepitantes } \\
\text { 4. } \uparrow \mathrm{R} \\
\text { 5. Tiraje } \\
\text { 6. } \mathrm{SaO}_{2}<90 \% \\
\text { 7. } \mathrm{PaOO}_{2}>45 \mathrm{mmHg} \\
\text { 8. } \mathrm{PaO}_{2}<60 \mathrm{mmHg}\end{array}$ \\
\hline Shaw et al., 19919 & 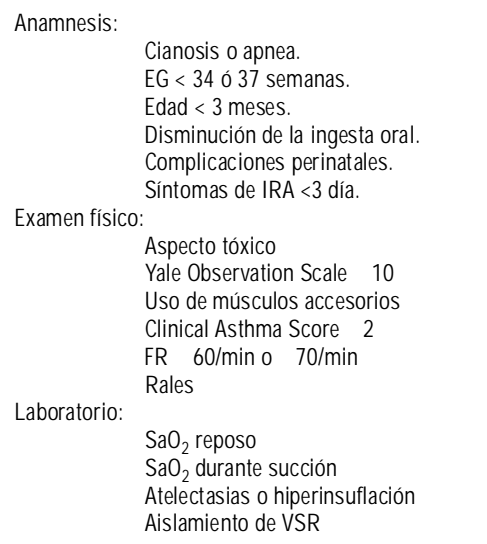 & $\begin{array}{l}\text { "Enfermedad leve" (alerta, activo y con tolerancia } \\
\text { alíquidos sin requerimiento de O2) vs. "enfermedad } \\
\text { severa". }\end{array}$ & $\begin{array}{l}\text { Datos clínicos y bioquímicos independientemente aso- } \\
\text { ciados con enfermedad severa por análisis multifacto- } \\
\text { rial: } \\
\text { 1. Aspecto tóxico. } \\
\text { 2. SaO2 }<95 \% \text {. } \\
\text { 3. EG }<34 \text { semanas. } \\
\text { 4. } \mathbb{R}>70 / \text { min. } \\
\text { 5. Edad }<3 \text { meses. }\end{array}$ \\
\hline Saijo et al., 199610 & $\begin{array}{l}\text { 1. Recuento de leucocitos }>15000 / \mathrm{mm} 3 \\
\text { 2. Recuento de neutrófilos }>10000 / \mathrm{mm} 3 \\
\text { 3. Eritrosedimentación }>30 \mathrm{~mm} / \mathrm{hora} \\
\text { 4. Proteina C reactiva }>3.0 \mathrm{mg} / \mathrm{dL} \text {. }\end{array}$ & $\begin{array}{l}\text { Hallazgos de neumonía lobar vs. bronconeumonía } \\
\text { vs. bronquiolitis en niños con IRAB por VSR hos- } \\
\text { pitalizados }\end{array}$ & $\begin{array}{l}\text { La prevalencia de los } 4 \text { indicadores fue mayor en los } \\
\text { pacientes con neumoníalobar comparados contrabron- } \\
\text { quiolitis o bronconeumonía }\end{array}$ \\
\hline
\end{tabular}

IRAB: infección respir atoria aguda baja. IRA: infección respir atoria alta. FR: :frecuencia respiratoria; $\mathbf{F C}_{\text {: frecuencia cardíaca. SaO }} \mathbf{2}$ : saturación de oxígeno. $\mathrm{PaO}_{2}$ :presión arterial de oxígeno. $\mathbf{P a C O}_{2}$ : presión ar terial de dióxido de carbono. ESD:eritrosedimentación. PCR: proteína C-reactiva.

\section{Hemogramas}

En 10 de los estudios revisados se solicitaron hemogramas en todos los pacientes. Sólo uno encontró mayor prevalencia de leucocitosis $(>15,000 / \mathrm{mm} 3)$ o neutrofilia $(>10,000 / \mathrm{mm} 3)$ en neumonías lobares versus bronquiolitis o bronconeumonía;ninguno corroboró la utilidad del hemograma para el diagnóstico o pronóstico de la bronquiolitis.

\section{Radiología de tórax}

Si bien en casi todos los estudios se realizaron placas de tórax con variable prevalencia de hallazgos como hiperinsuflación pulmonar, infiltrados difusos o atelectasias, sólo 2 examinaron específicamente la relación entre anormalidades radiológicas y severidad de la bronquiolitis. En uno se observó que los pacientes con atelectasias tuvieron 2.7 veces más riesgo de enfermedad severa (IC 95\%: 1.97-3.7), mientras que el otro no encontró asociación entre hallazgos radiológicos y severidad. En otro estudio ${ }^{6}$ las anomalías radiológicas se correlacionaron con el uso de antibióticos, cuya efectividad no se valoró (probablemente uso injustificado dada la etiología viral). En síntesis, la radiología de tórax puede ser útil para predecir casos severos pero no debe efectuarse en niños con enfermedad leve ya que no altera el manejo clínico.

\section{Diagnóstico virológico}

En la literatura analizada la prevalencia de VSR en bronquiolitis osciló entre el 26 y el 95\%.Para el diagnóstico virológico pueden realizarse tests específicos, como métodos de aislamiento viral, pruebas de detección rápida de antígenos como inmunofluorescencia directa (IFA) o inmunoenzimáticas (ELISA), reacciones de cadena de polimerasa (PCR) y títulos de anticuerpos en agudo o convalecientes. Estos tests tienen una sensibilidad del $80-90 \%$ pero su utilidad clínica es relativa, ya que no suelen cambiar conductas de manejo, excepto medidas de aislamiento o tratamiento antiviral específico en subgrupos de alto riesgo. Los principales resultados de estudios diagnósticos sobre tests virológicos se resumen en la TABLA 2. 
Tabla 2: Estudios diagnósticos sobre pruebas virológicas

\begin{tabular}{|c|c|c|c|}
\hline AUTOR & “GOLD STANDARD" & TESTS COMPARADOS & RESULTADOS \\
\hline Ahluwalia et al., 198711 & $\begin{array}{l}\text { Cultivo viral de muestras de secreciones naso- } \\
\text { faríngeas }\end{array}$ & $\begin{array}{l}\text { 日ISA, IFA sobre muestras de secreciones naso- } \\
\text { faríngeas }\end{array}$ & $\begin{array}{l}\text { 日IISA: } S=61-69 \% \quad E=100 \% \\
\text { IFA: } S=52-61 \% \quad E=78-89 \%\end{array}$ \\
\hline $\begin{array}{l}\text { Chattopadhya et al., } \\
1992^{12}\end{array}$ & Cultivo viral & IFA, 且ISA & 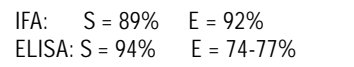 \\
\hline $\begin{array}{l}\text { Eugene-Ruellan et al., } \\
1998^{13}\end{array}$ & Cultivo viral y/o IFA & PCR & $97 \%$ de concordancia \\
\hline Ong et al., 200114 & IFA & PCR & IFA detectó 27 casos, PCR detectó 28 \\
\hline Waner et al., 199015 & Cultivo viral y/o IFA & 且ISA & $E=91 \%$ \\
\hline
\end{tabular}

ELISA: pruebas inmunoenzimáticas. IFA: inmunofluorescencia directa. PCR: reacción de cadena de polimerasa. S: sensibilidad; E: especificidad.

\section{RECOMENDACIONES DIAGNÓSTICAS}

En general, el diagnóstico de bronquiolitis se efectúa por anamnesis y examen físico, y los predictores de severidad son en su mayoría también clínicos. No existe evidencia que justifique el uso de exámenes complementarios en el diagnóstico de la enfermedad, excepto para diferenciarla de otras entidades (neumonía, insuficiencia cardíaca congestiva). La existencia de leucocitosis, neutrofilia y reactantes de fase aguda parece asociarse con mayor frecuencia a sobreinfección bacteriana pudiendo justificar el uso de antibióticos, aunque no hay evidencia sólida que sustente esta conducta. Además, los estudios diagnósticos para documentar el agente etiológico en IRAB no son útiles, o bien porque carecen de buena capacidad operativa (como el aspirado de secreciones naso-traqueales) o porque son demasiado cruentos para casos no severos (como el lavado broncoalveolar o los cultivos tisulares). La cuestión de real interés es si se debe buscar el VSR en todos los casos de bronquiolitis, ya que no cambia el manejo. Algunas instituciones requieren la identificación para cohortizar los casos intentando disminuir la transmisión intrahospitalaria, aunque la utilidad de esto no ha sido verificada. Se justifica investigar el VSR en niños con bronquiolitis si: (a) se desea evitar una batería de estudios para descartar sepsis en un lactante febril menor de 3 meses; (b) el diagnóstico clínico de bronquiolitis no está claro; (c) se requiere confirmar la etiología en una investigación de efectividad; (d) se efectúa vigilancia epidemiológica de IRAB en niños en el ámbito de la salud pública. Finalmente, no existen estudios aleatorizados que evalúen si realizar exámenes complementarios modifica el desenlace de la enfermedad.

\section{EFECTIVIDAD DE LAS INTERVENCIONES FARMA- COLÓGICAS TERAPÉUTICAS PARA BRONQUIOLITIS}

Un total de 52 estudios investigaron distintos fármacos para tratar la bronquiolitis, ya sea comparados contra placebo o entre sí. La evidencia sobre intervenciones terapéuticas para bronquiolitis se resume en la TABLA 3.

\section{Nebulizaciones con adrenalina versus placebo}

En un ECA doble ciego controlado con placebo ${ }^{16}$ se aleatorizó a 34 niños con bronquiolitis sin comorbilidad a ser nebulizados con adrenalina racémica o placebo de solución fisiológica (SF).El grupo tratado tuvo mejoría significativa en la saturometría $\left(\mathrm{SaO}_{2}\right)$ promedio y los scores clínicos en la primera hora (mayor si la oximetría basal era $<93 \%$ ). El estudio es pequeño y no analiza el impacto sobre otras variables como tiempo de internación, costos o persistencia de sibilancias.

\section{Adrenalina subcutánea versus placebo}

Para valorar la eficacia de la adrenalina subcutánea versus placebo se incluyeron en un $\mathrm{ECA}^{17} 30$ niños menores de 2 años no asmáticos con sibilancias. Se observó mejoría de $\geq 4$ puntos en el RACS en $56 \%$ del grupo experimental contra sólo $7 \%$ de los controles.No hubo efectos adversos.Dado que gran parte de los niños en ambos grupos tenía historia familiar de atopía o antecedentes de broncobstrucción (aunque sin broncodilatadores), por lo que podrían considerarse portadores de hiperreactividad bronquial, es probable que los resultados de este estudio no sean generalizables a poblaciones diferentes.

\section{Nebulizaciones con adrenalina versus nebulizaciones con broncodilatadores $\beta 2$ (salbutamol o albuterol)}

Se localizaron 4 ensayos aleatorizados y $\operatorname{ciegos}^{18}$ a 21 comparando nebulizaciones con adrenalina y con broncodilatadores $\beta 2$ (3 de salbutamol, 1 de albuterol) en menores de 2 años sin asma o comorbilidad. No hubo diferencias significativas en los puntos finales primarios (FR, FC, puntajes clínicos, requerimiento de O2, $\mathrm{SaO}_{2}$ y tiempo de internación), a excepción de un estudio donde el grupo adrenalina mostró mejoría significativa en la $\mathrm{SaO}_{2}$ a la hora y menor tasa de internación (33\% vs.81\%). No se observó rebote post-tratamiento, pero sí mayor taquicardia a las 48 horas y palidez a 30-60 minutos con el uso de adrenalina. Estos estudios son pequeños para detectar diferencias clínicamente relevantes, por lo que la evidencia actual es insuficiente.

Nebulizaciones con $\beta 2$ (salbutamol o albuterol) versus $\beta 2$ orales, bromuro de ipatropio, placebo o ningún tratamiento La eficacia de los broncodilatadores $\beta 2$ versus otras terapias se valoró en 11 estudios22 a 32, la mayoría en niños menores de 2 años (algunos hasta 6 años).Casi todos los resultados primarios fueron medidas de corto plazo y todas las diferencias significativas fueron halladas en la primera hora post-tratamiento.Se encontró mejoría significativa de los scores clínicos en los grupos con $\beta 2$ comparados con placebo en 3 estudios, y en otro respecto del puntaje basal (en los grupos albuterol y control). Como eventos adversos hubo mayor taquicardia asociada a $\beta 2$ en 3 estudios, y en otro una tendencia a hipoxemia progresiva en pacientes con albuterol lo que preocupa sobre la seguridad de dosis repetidas. Esta serie tiene los mismos problemas: pocos pacientes para detectar diferencias de trascendencia clínica, coexistencia de bronquiolitis con asmáticos o hiperreactivos, múltiples agentes, dosis y resultados, y falta de datos sobre efectos relevantes a mayor plazo. 
Tabla 3: Síntesis de la evidencia sobre tratamiento de la bronquiolitis.

\begin{tabular}{|c|c|c|c|c|c|c|}
\hline \multirow[t]{2}{*}{ TRATAMIENTOS } & \multicolumn{3}{|c|}{ MAGNITUD DE LA EVIDENCIA } & \multirow{2}{*}{$\begin{array}{l}\text { CALIDAD DE LOS } \\
\text { ESTUDIOS }\end{array}$} & \multirow{2}{*}{$\begin{array}{l}\text { MAYOR EVIDENCIA } \\
\text { A FAVOR DEL } \\
\text { TRATAMIENTO }\end{array}$} & \multirow{2}{*}{$\begin{array}{l}\text { EVENTOS ADVERSOS } \\
\text { EN GRUPO TRATADO }\end{array}$} \\
\hline & $\mathbf{N}^{0}$ de estudios & Tamaño muestral & $\mathrm{N}^{\circ}$ total de pacientes & & & \\
\hline $\begin{array}{l}\text { Nebulizaciones con adrena- } \\
\text { lina vs. placebo SF }\end{array}$ & 1 & 29 & 29 & Regular & $\begin{array}{l}\mathrm{SI} \text { (para } \mathrm{SaO}_{2} \text { y mejoría } \\
\text { de scores clínicos a los } \\
60 \text { ' postratamiento) }\end{array}$ & Palidez peribucal \\
\hline $\begin{array}{l}\text { Adrenalina parenteral vs. } \\
\text { Placebo }\end{array}$ & 1 & 30 & 30 & Buena & $\begin{array}{l}\text { SI (para cambios en } \\
\text { score respiratorio) }\end{array}$ & No reportados \\
\hline $\begin{array}{l}\text { Nebulizaciones con adrena- } \\
\text { lina vs. nebulizaciones con } \\
\beta 2 \text { (salbutamol o albuterol) }\end{array}$ & 4 & $24-100$ & 195 & \begin{tabular}{|l|} 
Buena (3) \\
Regular (1)
\end{tabular} & $\begin{array}{l}\text { NO (excepto } \mathrm{SaO}_{2} \text { a } 60 \\
\text { y tasa de internación en } \\
\text { estudio de Menon) }\end{array}$ & $\begin{array}{llll}\text { Ambas } & \uparrow F C & 0 & \downarrow F C \text {, } \\
\text { palidez } & & & \\
\end{array}$ \\
\hline $\begin{array}{l}\text { Nebulizaciones con } \beta 2 \\
\text { (salbutamol o albuterol) vs. } \\
\text { broncodilatadores orales, } \\
\text { bromuro deipatropio nebu- } \\
\text { lizado, placebo o nada }\end{array}$ & 11 & $21-169$ & 784 & $\begin{array}{l}\text { Excelente (2) } \\
\text { Buena (5) } \\
\text { Regular (4) }\end{array}$ & $\begin{array}{l}\text { SI (para scores clínicos } \\
\text { a30-60' postratamiento } \\
\text { y cambios en puntajes } \\
\text { clínicos) }\end{array}$ & $\begin{array}{l}\text { Tendencia a hipoxiay dis- } \\
\text { tress respiratorio, } \uparrow F C \\
\text { significativo }\end{array}$ \\
\hline $\begin{array}{l}\text { Nebulizaciones con } \beta 2 \text { vs. } \\
\text { bromuro de ipatropio vs. ca- } \\
\text { da uno aislado y/o placebo }\end{array}$ & 3 & $62-89$ & 220 & \begin{tabular}{|l} 
Buena (2) \\
Regular (1)
\end{tabular} & NO & Temblores, $\uparrow F C$ \\
\hline $\begin{array}{l}\text { Corticoides orales vs. place- } \\
\text { bo, con o sin broncodilata- } \\
\text { dores }\end{array}$ & 7 & $28-114$ & 406 & $\begin{array}{l}\text { Excelente (2) } \\
\text { Buena (4) } \\
\text { Regular (1) }\end{array}$ & $\begin{array}{l}\text { SI (para internación, fra- } \\
\text { caso terapéutico y } \\
\text { scores clínicos) }\end{array}$ & $\begin{array}{l}\text { Generalmente no reporta- } \\
\text { dos o no relacionados a } \\
\text { la intervención }\end{array}$ \\
\hline $\begin{array}{l}\text { Dexametasona parenteral vs. } \\
\text { placebo }\end{array}$ & 2 & $29-118$ & 147 & $\begin{array}{l}\text { Buena (1) } \\
\text { Regular (1) }\end{array}$ & NO & $\begin{array}{l}\text { Sangre oculta en materi- } \\
\text { al fecal con dexametasona }\end{array}$ \\
\hline $\begin{array}{l}\text { Corticoides nebulizados vs. } \\
\text { placebo o tratamiento con- } \\
\text { vencional }\end{array}$ & 6 & $40-161$ & 492 & $\begin{array}{l}\text { Buena (3) } \\
\text { Regular (2) } \\
\text { Pobre (1) } \\
\end{array}$ & $\begin{array}{l}\text { Alguna evidencia a favor } \\
\text { en outcomes alargo pla } \\
\text { zo (9 semanas a } 1 \text { año) }\end{array}$ & $\begin{array}{l}\text { Candidiasis oral, empeo- } \\
\text { ramiento de síntomas en } \\
\text { grupo tratado }\end{array}$ \\
\hline Ribavirina vs. placebo & 7 & $19-41$ & 212 & $\begin{array}{l}\text { Excelente (1) } \\
\text { Buena (2) } \\
\text { Regular (4) }\end{array}$ & NO & $\begin{array}{l}\text { Eritema de párpados tran- } \\
\text { sitorio, distress respira- } \\
\text { torio agudo }\end{array}$ \\
\hline $\begin{array}{l}\text { Antibióticos vs. nadau otros } \\
\text { antibióticos }\end{array}$ & 2 & $61-233$ & 294 & $\begin{array}{l}\text { Regular (1) } \\
\text { Pobre (1) }\end{array}$ & NO & $\begin{array}{l}\text { No reportados para sub- } \\
\text { grupo con bronquiolitis }\end{array}$ \\
\hline $\begin{array}{l}\text { Tratamiento con RSVIGen- } \\
\text { dovenosa }\end{array}$ & 2 & $98-98$ & 196 & $\begin{array}{l}\text { Excelente (1) } \\
\text { Buena (1) }\end{array}$ & NO & $\begin{array}{l}\text { Sobrecarga hídrica, dis- } \\
\text { tress respiratorio (ambos } \\
\text { grupos) }\end{array}$ \\
\hline $\begin{array}{l}\text { Otras intervenciones tera- } \\
\text { péuticas }\end{array}$ & 6 & $13-96$ & 274 & $\begin{array}{l}\text { Buena (3) } \\
\text { Regular (3) }\end{array}$ & Resultados variables & Reportes variables \\
\hline
\end{tabular}

RSVIG: Inmunoglobulina específica para VSR FC: Frecuencia Cardíaca FR: Frecuencia Respiratoria Sa0 2 : Saturometría

\section{Nebulizaciones con $\beta 2$ (salbutamol o albuterol) + bromuro de ipatropio combinados versus aislados $\mathrm{y} / \mathrm{o}$ placebo}

La combinación de broncodilatadores $\beta 2$ y bromuro de ipatropio se evaluó en 3 estudios 33 a 35. No se encontraron ventajas para ninguna rama de tratamiento en ninguno de los outcomes primarios (FR, scores clínicos o tiempo de internación) Nuevamente hubo mayor taquicardia con el uso de albuterol. Sin embargo, los grupos son pequeños y en uno de los estudios hubo exclusiones postrandomización, por lo que se requieren nuevos estudios para enunciar recomendaciones válidas.

\section{Corticoides orales versus placebo, con o sin broncodi- latadores asociados}

Seis estudios compararon corticoides orales (prednisolona, prednisona o dexametasona) a placebo 36 a 41 , utilizando como cointervención broncodilatadores de base o según necesidad.Se observaron pocas diferencias en scores clínicos, admisión o reingreso, persistencia de síntomas o necesidad de otros tr atamientos. En los grupos con corticoides, se informó en distintos estudios mejor respuesta clínica, menor tasa de internación (19\% vs.44\%), mejores puntajes clínicos a las 4 horas y menor necesidad de corticoterapia al alta.En un tercio de los pacientes de un ECA se encontró persistencia de síntomas respiratorios a los 2 años, algo mayor en el grupo que recibió prednisona.En dos informes secuenciales se reportó que los corticoides mejoraron la respuesta clínica en pacientes no ventilados y acortaron la internación en ventilados, pero que luego de un seguimiento de 5 años no hubo diferencias significativas en desenlaces a largo plazo.No se informaron efectos adversos de la corticoterapia. Si bien estos estudios tienen las mis- mas limitaciones ya mencionadas, se midieron algunos resultados de interés como internación y desarrollo de asma, pero la mayoría de las diferencias se halló en variables menos relevantes.

\section{Corticoides parenterales versus placebo}

Esta comparación fue analizada en 2 estudios ${ }^{42,43}$. En una muestra de 122 niños menores de 1 año con bronquiolitis a los que se aleatorizó a recibir dexametasona intramuscular durante 3 días o placebo, no se encontraron diferencias significativas en el tiempo de oxigenoterapia o de mejoría clínica (es posible que se haya subestimado el efecto dado que el grupo tratado tenía $<\mathrm{SaO}_{2}$ ). El único evento adverso descripto fue sangre oculta en materia fecal (en ambos grupos). En otro grupo de 32 niños menores de 2 años a los que se randomizó a recibir placebo o una dosis de carga de dexametasona seguida de 2 días de mantenimiento, tampoco hubo diferencias en tiempo de internación u otras variables de resultado. No hubo efectos adversos. Esta evidencia es insuficiente para sustentar que los corticoides parenterales tengan utilidad en la bronquiolitis.

\section{Corticoides nebulizados versus placebo o tratamiento ha- bitual}

Se encontraron 6 estudios de budesonide 44 a 49 y 1 de fluticasona 50 por vía inhalatoria, comparados contra placebo o tratamiento habitual $\left(\mathrm{O}_{2}\right.$, broncodilatadores y/o adrenalina). El budesonide se relacionó en distintos ECA con menor necesidad de terapia inhalatoria a los 2 años, menor persistencia de sibilancias a mediano y largo plazo y menos fracasos terapéuticos que el placebo, pero no hubo diferencias con otros grupos de tratamiento ac- 
tivo (cromoglicato sódico inhalado, terbutalina inhalatoria y prednisolona oral). En cambio, en otros 2 ECA el budesonide se asoció con un número mayor de reinternaciones por problemas respiratorios (48\% versus 10\%) y episodios sintomáticos al año (validez comprometida por pérdida al seguimiento del $20 \%$ ). El uso de fluticasona se asoció con menos episodios nocturnos de tos, mejores tests funcionales respiratorios a las 36 semanas, y con 2 casos de candidiasis oral como eventos adversos. Estos estudios pequeños tuvieron las mismas falencias que los anteriores, pero la edad promedio fue menor, y hubo efectos de relevancia clínica a mediano y largo plazo.La evidencia específica en bronquiolitis es aún conflictiva para recomendar el uso de corticoides inhalatorios.

\section{Ribavirina versus placebo}

La eficacia de la ribavirina aerosolizada contra placebo para tratar la bronquiolitis por VSR en niños menores 6 meses se investigó en 7 estudios pequeños 51 a57.La ribavirina se asoció a mejoría clínica y menos episodios de sibilancias a los 6 años (esto podría estar sobrestimado debido a que el grupo control tuvo mayor gravedad y más pérdidas al seguimiento). Como eventos adversos se reportaron un episodio de eritema de párpados transitorio y un caso de distress respiratorio agudo que llevó a la suspensión.La falta de evidencia sobre efectos relevantes ya había sido informado en un metanálisis 58 .

\section{Antibióticos}

Ningún estudio evaluó la eficacia del tratamiento antibiótico en bronquiolitis, aunque 2 trabajos incluyeron casos de bronquiolitis como subgrupo entre IRAB 59,60.Ninguna evidencia en estos estudios sustenta la eficacia de los antibióticos en la bronquiolitis, excepto en los casos complicados por sobreinfección bacteriana o algunos con otitis media concomitante.

\section{Inmunoglobulina específica para VSR (RSVIG)}

El uso terapéutico de RSVIG en bronquiolitis por VSR fue evaluado en niños previamente sanos61y en pacientes con alto riesgo de complicaciones (prematuros o patología cardio-pulmonar menores de 2 años) ${ }^{62}$.En ninguno de los 2 estudios hubo ventajas en tiempo de internación, cuidados intensivos o ARM, necesidad de $\mathrm{O}_{2} \mathrm{u}$ otras medicaciones, scores, infección por VSR o reinternación en la temporada siguiente.En ambos casos, se requiere más evidencia para recomendar su uso terapéutico.

\section{Otras intervenciones terapéuticas}

Varios estudios evaluaron la eficacia de otras terapias para bronquiolitis: alfa-2 interferon ${ }^{63}, \mathrm{O} 2$ con helio ${ }^{64}$, surfactante porcino ${ }^{65}$ aerosol de furosemida66y desoxi-ribonucleasa recombinante humana67. La evidencia es insuficiente para sustentar su eficacia, excepto la administración de helio y surfactante que evidenciaron algunas ventajas clínicas en bronquiolitis severas que deberían verificarse en estudios grandes bien diseñados.

\section{RECOMENDACIONES TERAPÉUTICAS}

El tratamiento de la bronquiolitis es principalmente sintomático y de sostén.Las recomendaciones de la American Academy of Pediatrics $^{68}$ del año 2000 incluían medidas de sostén (hidratación, oxígenoterapia y eventualmente ventilación mecánica) desaconsejando el uso de corticoides o antimicrobianos.El único tratamiento específico es la ribavirina para pacientes de riesgo con enfermedad severa documentada por VSR. El uso de broncodilatadores $\beta 2$ y corticoides es una práctica difundida a pesar de los datos conflictivos sobre su verdadera efectividad. Para ninguna de la intervenciones terapéuticas analizadas existe evidencia consistente, sobre todo en relación a resultados clínicamente relevantes o im- pacto a mediano o largo plazo. Para algunas parece haber beneficios potenciales que deberían evaluarse en ECA de buen tamaño y diseño comparados contra placebo o tratamiento convencional: nebulizaciones con adrenalina, salbutamol y/o bromuro de ipatropio, corticoides orales o parenterales (dexametasona) o inhalados (budesonide).Intervenciones como la helioterapia o el surfactante deben reservarse para pacientes severos o ventilados. La dudosa efectividad y los efectos adversos potenciales impiden recomendar el uso terapéutico de ribavirina, antibióticos, RSVIG, fursemida, desoxirribonucleasa o alfa interferón, excepto en el contexto de estudios experimentales. El budesonide debe usarse con precaución debido a los efectos adversos descriptos. En síntesis, en base a esta revisión ninguna intervención farmacológica para el tratamiento de la bronquiolitis parece ser más efectiva como para ser recomendada.Otros metanálisis publicados aportan evidencia adicional sobre la efectividad en bronquiolitis de broncodilatadores 69,70 y corticoides ${ }^{71}$ Respecto de los $\beta 2$ agonistas, un metanálisis que analizó 8 ECA concluye que no hay suficiente evidencia para recomendar su uso, mientras que otro que incluyó 15 ECA informa una mejoría modesta a corto plazo en bronquiolitis no severas: reducción en puntajes promedio (-0.32 puntos; IC 95\%: 0.54 a $-0.11 ; p<0.01)$ y mejoría clínica ( $R R=0.76$; IC 95\%: 0.60$0.95 ; p=0.02)$ aunque sin efecto sobre la hospitalización ( $R R=0.85$; IC 95\%:0.47-1.53; $p=0.58)$. En cuanto a los corticoides, un metanálisis de 6 estudios, reportó ventajas a favor del tratamiento:mejores puntajes clínicos (-1.60 puntos; IC 95\%: -1.92 a -1.28) y menor tiempo de internación (-0.43 días; IC 95\%: -0.81 a -0.05), concluyendo que su uso debería ser considerado en la bronquiolitis.

\section{EFECTIVIDAD DE LA PROFILAXIS EN LA PREVENCIÓN DE BRONQUIOLITIS}

\section{RSVIG endovenosa versus placebo o tratamiento habitual para prevenir bronquiolitis por VSR}

Se localizaron 4 estudios evaluando el uso de inmunoglobulina específica para prevenir la bronquiolitis por VSR en niños de riesgo normal o alto. El estudio PREVENT ${ }^{72}$ es un ensayo clínico multicéntrico que randomizó a 510 niños menores de 2 años de alto riesgo (displasia broncopulmonar-DBP- o prematurez) a recibir RSVIG o placebo de albúmina durante la temporada de VSR. EI tratamiento se asoció a una reducción cercana al $50 \%$ en los distintos outcomes analizados: internaciones por VSR ( $8 \%$ vs. $13.5 \%)$ o enfermedad respiratoria ( $16 \%$ vs. $27 \%$ ), días de hospital por VSR o por enfermedad respiratoria (170 vs. $317 / 100$ niños), días con $\mathrm{O}_{2}$ (34 vs. 85/100 niños) y con scores severos (49 vs. $106 / 100$ niños). Hubo también más suspensiones o retardos de la infusión por eventos adversos en el grupo experimental ( $3 \%$ vs. $1 \%)$. Resultados consistentes se encontraron en otros 2 estudios $^{73,74}$ en niños de alto riesgo menores de 4 años, que informaron reducción en los casos de IRAB por VSR, las admisiones y días de internación o de cuidados intensivos, incluso en la temporada siguiente, sin eventos adversos. La RSVIG administrada en forma endovenosa mensual durante la temporada pico de VSR parece ser útil para prevenir la enfermedad en niños de alto riesgo. Sin embargo, su uso clínico ha sido superado por el palivizumab.

\section{Anticuerpos monoclonales para la profilaxis de la bron- quiolitis por VSR}

El palivizumab es un anticuerpo monoclonal que provee inmunidad pasiva tipo IgG contra la infección por VSR cuando es administrada previa al pico epidemiológico. Esta intervención profiláctica fue evaluada por un único gran ECA controlado con placebo:el estudio IMPACT-RSV75, que incluyó 1502 niños de alto riesgo (pre- 
maturos menores de 6 meses, DBP menores de 24 meses) a los que se randomizó a recibir palivizumab o placebo cada mes durante 5 meses. La profilaxis redujo significativamente la tasa de hospitalización por VSR (4.8\% vs.10.6\%), el tiempo de internación (36.4 vs. 62.6 días/100 niños), de oxigenoterapia (30.3 vs. 50.6 días/100 niños) y de puntajes severos (29.6 vs.47.4 días/100 niños), y los requerimientos de cuidados intensivos (1.3\% vs. 3\%). Estas diferencias se verificaron sólo para las infecciones por VSR y para todos los subgrupos de riesgo. Los efectos adversos (fiebre, irritabilidad, diarrea y reacción en el sitio de inyección) fueron similares al grupo placebo. El palivizumab es una intervención profiláctica efectiva y segura para prevenir la enfermedad severa por VSR en grupos de alto riesgo, y su administración por vía intramuscular es más fácil que la de RSVIG que requiere acceso endovenoso. La información sobre otro anticuerpo monoclonal (SB $209763)^{76}$ es aún insuficiente para recomendar su uso.

\section{Vacunas para la prevención de bronquiolitis por VSR}

Se localizaron 3 estudios pequeños sobre la eficacia de vacunas para prevenir la infección en niños de alto riesgo 77a79. En un ECA de 21 niños menores de 12 meses con DBP e infección previa por VSR, la vacunación se asoció a una reducción no significativa en las infecciones por VSR. En niños con fibrosis quística, 2 estudios (uno contra placebo y otro comparando la administración secuencial anual versus una única dosis) informaron significativamente menos episodios de IRAB y días de enfermedad en los vacunados. La baja potencia y falencias de diseño impide extraer conclusiones válidas como para recomendar la vacuna, aunque parece ser promisoria en niños con alto riesgo por prematurez, DBP o fibrosis quística.

\section{RECOMENDACIONES PREVENTIVAS}

Aunque la bronquiolitis suele ser un cuadro autolimitado en la mayoría de los casos, puede tener complicaciones serias y alta mortalidad en niños con historia de prematurez o comorbilidades (enfermedad cardíaca o respiratoria crónica como DBP).La profilaxis previa a la temporada invernal tanto con palivizumab como con inmunoglobulina específica para VSR han demostrado ser intervenciones efectivas en estas subpoblaciones de alto riesgo (prematuros menores de 32 semanas de edad gestacional con enfermedad pulmonar crónica). La extensión de la recomendación de su uso a otros subgrupos (como los niños cardiópatas) requiere de nueva evidencia, lo mismo que la inmunización activa con vacunas que aún están en desarrollo.

\section{COSTO-EFECTIVIDAD DE LA PROFILAXIS PARA BRON- QUIOLITIS}

No obstante la efectividad del palivizumab para prevenir la enfermedad por VSR en pacientes de alto riesgo, su uso en poblaciones menos restringidas ha sido limitado debido a dudas acerca de su costo-efectividad. Para objetivar medidas (como el costo adicional por internación evitada) que interesan para tomas de decisión sanitarias, se revisó la información de 6 estudios de evaluación económica ${ }^{80 a 85}$ sobre el uso de palivizumab en distintas poblaciones de riesgo.En líneas generales, estos estudios tomaron como base al estudio IMPACT para la estimación de las tasas de hospitalización (10.6-42.6\%) y su reducción mediante la profilaxis (55\%). Los costos de cada internación por VSR fueron estimados de la literatura o de centros de internación (en USA, Australia y Argentina) y oscilaron entre U\$S 10.000 y 166.000.El costo del palivizumab varió entre U\$S 2.500 y 4.500 por niño por temporada. Un único estudio incluyó la perspectiva de la sociedad tomando en cuen-

\section{Dra. Graciela Demirdjian [ Hospital Garraham ]}

ta no sólo los costos médicos sino también los incurridos en concepto de viáticos, pérdida de días de trabajo de los padres y productividad futura por mortalidad precoz;en este estudio, la pérdida de tiempo de trabajo de los padres fue estimada en U\$S 44 por cada dosis de palivizumab y U\$S 358 por cada hospitalización, y el costo del tratamiento de una bronquiolitis se calculó en U\$S 198 si fue ambulatorio y U\$S 8.502 si cursó internación. En un estudio en el que se compararon las tasas de hospitalización antes y después de haber implementado la profilaxis obligatoria con palivizumab en un estado americano, la hospitalización por VSR se redujo al $1.6 \%$ en la etapa post-implementación respecto del control histórico del $25 \%$ del período previo, concluyendo que la profilaxis generó ahorros significativos. En Argentina, Fariña y col realizaron un análisis regional de costo-efectividad del palivizumab en niños de alto riesgo: 42 pacientes fueron seguidos por un período de 2 años, estimando una tasa de internación de $23.8 \%$, un costo promedio de $\$ 18.477$ por internación y de $\$ 1.100$ por dosis de palivizumab por paciente, y de $\$ 15.358$ por cada internación evitada.

La evidencia analizada permite conjeturar que la profilaxis con palivizumab en niños de 32-35 semanas de edad gestacional podría generar desde un ahorro de U\$S 36.040 por niño hasta un costo adicional máximo de U\$S 328.000 por hospitalización evitada (promedio de U\$S 50.000).La profilaxis se mostró más costo-efectiva en subgrupos de máximo riesgo (Edad gestacional 2332 semanas con 1 mes de oxigenoterapia y alta en época invernal).

\section{CONCLUSIONES SOBRE LA COSTO-EFECTIVIDAD DE LA PROFILAXIS}

Los estudios sintetizados varían ampliamente en sus enfoques y en los índices de costo-efectividad obtenidos. Esto se explica porque éstos son generalmente muy sensibles a variaciones en las estimaciones utilizadas para calcularlos (tasas de hospitalización, impacto de la intervención o costos asociados a la profilaxis y al uso de recursos sanitarios). La mayoría de los estudios concluye que los costos de la profilaxis son ampliamente justificados por los ahorros potenciales. No obstante, la costo-efectividad no es el único factor a tener en cuenta para decidir el uso de una intervención sanitaria. Aspectos como la perspectiva desde la cual se analizan los costos, o el costo de oportunidad de asignar recursos a la profilaxis en diferentes contextos sanitarios, deben tenerse en cuenta para la toma de decisión en cuanto a su implementación.

\section{PUNTOS CLAVE DE ESTA REVISIÓN:}

- La bronquiolitis es una de las enfermedades de mayor impacto en la edad pediátrica, sobre todo en niños pequeños, prematuros o con comorbilidad cardio-respiratoria.

- El diagnóstico de bronquiolitis y la valoración de su severidad se basan en parámetros clínicos. No están indicados los exámenes de laboratorio, rayos o pruebas virológicas salvo en situaciones específicas.

- El tratamiento principal es el de sostén.No hay evidencia sólida que respalde el uso de broncodilatadores b2 agonistas, corticoides, adrenalina u otras intervenciones farmacológicas.

- La profilaxis con palivizumab en niños de alto riesgo de infección por VSR (prematuros, pacientes con enfermedad pulmonar crónica o cardiópatas) es una intervención de eficacia probada aunque de variable costo-efectividad.

- Se necesitan nuevos estudios aleatorizados de calidad que evalúen la eficacia de las distintas intervenciones terapéuticas en bronquiolitis sobre variables de resultados relevantes a mediano y largo plazo. 


\section{Bibliografía Recomendada}

1 Maragement of Bronchiolitis inInfants and Children". Evidence ReportTechnology Assessment No69. Agency Healthcare Research \& Quality. Texto completo: http://www.ahrq.gov/cliniclevrptfiles.htm\#bronch. Síntesis: http://ahopr.gov/dinic/epsum/broncsum.htm

2 Engle S, Newns GH. Proliferative mural bronchiolitis. Arch Dis Child. 1940;15:219.

3 Court SD. The definition of acute respiratory illnesses in children. Postgrad Med J. 1973;49:771-776.

4 Demy FW, QydeWA Aautelower respiratory tract infedions in norhospitalized children. J Pediatr. 1986; 108:635646.

$5 \mathrm{Tal}$ A, Ravilski G, Yoha Det al.Dexamethasoneand salbutamol in the treatment of acute whezing in infants. Pediatrics 1983;71: 13 .

6 Cherian T, Steinhoff MC, Simoes EA, John TJ. Cinical signs of acute lower respiratory tract infections inmanourished infants and children. Pediatr Infect Dis J. 1997;16:490-494.

7 Dawson KP, Long A, Kennedy J, Mogridge N The chest radiograph in acute bronchiolitis. J Paediatr Child Health 1990;26:209-211.

8 Muholland EK, Cinsky A, Shann FA Ginical findings and severity of autebronchiditis. Lancet. 1990;335:12591261.

9 Shaw KN, Bell LM, Sherman NH. Outpatient assessment of infants with bronchiolitis. Am J Dis Child 1991:145:151-155.

10 Sajo M, Ishii T, Kokubo M, Murono K, Takimoto M, Fujita K White blood œell count, Greactive protein and erythrocytesedimentation ratein respiratory syncytial virus infection of thelower respiratory tract. ActaPæe diatr Jpn. 1996;38:596-600.

11 Ahluwalia G Embree J, McNcol P, Law B, Hammond GN. Comparison of nasopharynged aspirate and na sopharyngeal swab specimens for respiratory syncytial virus diagnosis by cell culture, indirect immunofluorescence assay, and enzymelinked immunosorbent assay. J đin Microbiol. 1987;25:763-767.

12 Chattopadhya D, Chatterje R, Anand WK, Kumari S, Patwari AK. Lower respiratory tract infection in hospitalized children dueto respiratory syncytial (RS) virus during asuspected epidemic period of RSvirus in Ddhi. J Trop Pediatr. 1992;38:68-73.

13 Eugene-Ruellan G Freymuth F, Bahloul C, Badrane H, Vabret A, Tordo N Detection of respiratory syncytia virus $A$ and $B$ and parainfluenzavirus 3 sequences in respiratory tracts of infants by asingle $P O R$ with primers targeted to the L-polymerase ane and differential hy bridization. J Gin Microbiol. 1998;36:796-801.

14 Ong GM, Wyatt DE, ONeill HJ, McCaughey G, Coyle PV. A comparison of nested polymer ase chain reaction and immunofluorescence for thediagnosis of respiratory infections in children with bronchiolitis, and theimand immunofluorescencefor the diagnosis of respiratory infections i
plications for a cohorting strategy. J Hosp Infect. 2001:49:122-128.

15 Waner JL, Whitehurst NU, Todd SJ, Shalaby H, Wall LV. Comparison of directigen RSV with viral isolationand direct immunofluorescencefor theidentification of respiratory syncytial virus. J ain Microbiol. 1990;28:480483

16 Kristjansson S, Lodrup Carlsen KG, Wennergren G Strannegard IL, Carlsen KH Nebulised racemic adrena line in the treatment of acute bronchiolitis in infants and toddllers. Arch Dis Child. 1993;69:650-654.

7 Lowell Dl, Lister G Von Koss H, McCarthy P. Wheezing in infants: the response to epinephrine. Pediatrics. 1987;79:939-945.

18 Menon K Sutdiffe T, Kassen TP. A randomized trial comparing the efficacy of epinephrine with salbutamo in the treatment of aute bronchiolitis. J Pediatr. 1995;126:1004-1007.

19 Bertrand P, Aranibar H, Castro E Sanchez I. Eficacy of nebulized epinephrine versus salbutamol in hospitaized infants with bronchiolitis. Pediatr Pulmonol. 2001;31:284-288

20 Reijonen T, Korppi M, Pitkakangas S, Tenhola S, Remes K The clinical efficacy of nebulized racemic epinephrine and albuterol in acute bronchiolitis. Arch Pediatr Adolesc Med. 1995;149:686-692.

21 Sanchez I, De Koster J, Powell RE Wolstein R, Chernick V. Efect of racemic epinephrine and salbutamol on Sanchez I, De Koster J, Powell RE, Wolstein R, Chernick V. Efect of racemic epinephrine and salbu
dinicd score and pulmonary mechanics in infants with bronchiolitis. J Pediatr. 1993;122:145-151

dinicd score and pulmonary mechanics in infants with bronchiolitis. J Pediatr. 1993;122:145-151
22 Wassen TP, Rowe PC, Sutcliffe T, Ropp LJ, McDowell IW, Li MM. Randomized trial of salbutamol in acute Kassen TP, Rowe PC, Sutcliffe T, Ropp LJ,
bronchiolitis. J Pediatr.1991:118:807-811

23 Can D, Inan G Yendur G Oral R, Gunay I. Salbutamol or mist in acute bronchiolitis. Acta Paediatr Jpn 1998;40:252-255.

24 Dobson JV, Stephens-Groff SM, McMahon SR, Stemmler MM, Brallier SL, Bay C. The use of albuterol in hospitalized infants with bronchiolitis. Pediatrics. 1998;101:361-368.

25 Schuh S, Canny G Reisman W, e a d. Nebulized albuterol in acute bronchiolitis. J Pediatr. 1990;117:633-637.

26 Schweich PJ, Hurt TL, Wakkey $\mathrm{日}$, Mullen N, Archibald LF. The use of nebulized albuterol in wheering infants. Pediatr Emerg Care. 1992;8: 184-188.

27 Hckey RW, Gochman RF, Chande V, Davis HW. Albuterol delivered via metered-dose inhaler with spacer for outpatient treatment of young children with whœezing. Arch Pediatr Adolesc Med. 1994;148:189-194

28 Cengizier R, Saracla Y, Adalioglu G, Tuncer A. Effect of ord and inhaled sabutamol in infents with bronchiolitis.Acta Paediatr Jpn. 1997;39:61-63.

29 Gadomski AM, Lichenstein R, Horton L, King J, Keane V, Permutt T. Eficacy of albuterol in themanagement Gadomski AM, Lichenstein R, Horton L, Kng
of bronchiolitis. Pediatrics. 1994;93:907-912.

30 Gadomski AM, Aref GH, el Din CB, el Samy IH, Khallaf N, Black RE Oral versus nebulized albuterol in theman agement of bronchiolitis in Egypt. J Pediatr. 1994;124:131-138.

agement of bronchiolitis in Egypt. J Pediatr. 1994;124:131-138.
31 Goh A. Chay OM, Foo AL, Ong EK Efficacy of bronchodilators in the treatment of bronchiolitis. Singapore Med J.1997:38:326-328.

Med J.1997:38:326-328.

$32 \mathrm{HoL}$, Collis G Landau LI, Le Souef PN. Efect of sabutamol on oxygen saturation in bronchiolitis. Arch Dis Child 1991;66:1061-64

33Chowdhury D, al Howæi M, Knali M, al-Frayh AS, Chowdhury S, RamiaS. The role of bronchodilators in the management of bronchiolitis: aclinical tria. Ann Trop Paediatr. 1995;15:77-84.

34 Schuh S, Johnson D, Canny G et al. Efficacy of adding nebulized ipratropium bromide to nebulized albutero therapy in acute bronchiolitis. Pediatrics. 1992;90:920-923.

35 Wang E, Miner R, Allen U, Maj H. Bronchodilators for treatment of mild bronchiolitis: afactorial randomised trial. Arch Dis Child. 1992:67:289-293.

36 Goebel J, Estrada B, QuinonezJ, Nagii N, Sanford D, Boerth RC Prednisolone plus albuterol versus albuterol done in mild to moderate bronchiolitis. Ain Pediatr. 2000;39:213-220.

37 van Woensel JB, Kimpen JL, Sprilkelman AB, Oiwehand A van Aalderen WM. Long-term effects of predrisolonein the acutephaseof bronchiolitis caused by respiratory syncytial virus. Pediatr Pulmonol. 2000:30:9296.

38 van Woensel JB, Wolfs TF, van Aalderen WM, Brand PL, Kimpen JL. Randomised doubleblind placebo controlled trial of prednisolonein children admitted to hospital with respiratory syncytial virus bronchiolitis. Thorax. 1997;52:634-637.

39 Klassen TP, Sutcliffe T, Watters LK, Wells GA, Allen UD, L MM. Dexamethasone in salbutamol-treated inpatients with acute bronchiolitis: arandomized, controlled tria. J Pediatr. 1997;130:191-196.

40 Berger I, Argaman Z, Schwatz SB, et al. Eficacy of corticosteroids in acute bronchiolitis: short-term andlongterm follow-up. Pediatr Pulmonol. 1998:26:162-166.

42 Roosevelt G, Sheehan K, Gupp-Phelan J, TanzRR, Listernick R. Dexamethasone in bronchiolitis: a randomised controlled trial. Lancet. 1996;348:292-295.

43 DeBoeckK, Vander AaN Van LierdeS, Corbed L, Eeckels R. Respir đory syncytial virus bronchiolitis: adouble blind dexamethasone efficacy study. J Pediatr. 1997;131:919-921

44 Cade A, Brownlœ KG Conway SP, et al. Randomised placebo controlled trial of nebulised corticosteroids in acute respiratory syncytial vira bronchiolitis. Arch Dis Child. 2000;82:126-130.
45 Daugbjerg $\mathrm{P}$, Brenoe $\mathrm{E}$ Forchhammer $\mathrm{H}$, et d. A comparison between nebulized terbutaline, nebulized corticosteroid and systemic $\infty$ rticoster oid for acute wheering in children up to 18 months of age. Acta Paediatr. 1993; 82:547-51.

46 Fox GF, Everard ML, Marsh MJ, Milner AD. Randomised controlled trial of budesonide for the prevention of post-bronchiolitis wheezing. Arch Dis Child. 1999;80:343-347.

47 Kajosaari M, Syvanen P, Forars M, Juntunen-Backman K Inhaled corticosteroids during and after respiratory syncytial virus-bronchiolitis may decrease subsequent asthma Pediatr Allergy Immunol. 2000;11:198-202.

48 Rejonen T, Korppi M, KuikkaL, Remes K Anti-inflammatory therapy reduces wheezing after bronchiolitis. Arch Pediatr Adolesc Med. 1996;150:512-517.

49 Richter $\mathrm{H}$, Seddon P. Early nebulized budesonide in the treatment of bronchiolitis and the prevention of postbronchiolitic wheezing. J Pediatr. 1998;132:849-853.

50 Wong JY, Moon S, Beardsmore C, OCallaghan C, Simpson H. Nb objective benefit from steroids inhaled viaa spacer in infants recovering from bronchiolitis. Eur Respir J. 2000;15:388-394.

51 RodriguezWJ, Kim HW, Brandt $\mathrm{C}$, et al. Aerosolized ribavirin in the treatment of patients with respiratory syncytial virus disease. Pediatr Infect Dis J. 1987;6:159-163.

52 Taber LH, Knight V, Glibert BE, et al. Ribavirin aerosol treatment of bronchiolitis associated with respiratory syncytial virus infection in infants. Pediatrics. 1983;72:613-618.

53 Barry W, Cockburn F, Cornall R, Price JF, Sutherland G Vardag A Rbavirinaerosol for acutebronchiolitis. Arch Dis Child. 1986:61:593-597.

54 Everard ML, Swarbrick A, Rigby AS, Milner AD. The effect of ribavirin to treat previously heathy infants admitted with acute bronchiolitis on acute and chronic respiratory morbidity. Respir Med. 2001;95:275-280.

55 Guerguerian AM, Gauthier M, Lebel MH, Farrell CA, Laco oix J. Ribavirin in ventilated respiratory syncytial virus bronchiolitis. Arandomized, placebo-controlled tria. Am J Respir Oit Care Med. 1999;160:829-834.

56 Jana $\mathrm{H}$, Stutman HR, ZaleskaM, et al. Ribavirin effect on pulmonary function in young infants with respira tory syngytial virus bronchiolitis. Pediatr Infect Dis J. 1993;12:214-218.

57 Podriguez WJ , Arrobio J, Fnk R, Km HW, Milburn C. Prospective follow-up and pulmonary functions froma placebo-controlled randomized trial of ribavirin therapy in respiratory syncytial virus bronchiolitis. Ribavirin Study Group. Arch Pediatr Adolesc Med. 1999;153:469-474.

58 Randolph AG Wang EI Ribavirin for respiratory syncytial virus lower respiratory tract infection. Asystematic overview. Arch Pediatr Adolesc Med. 1996;150:942-947.

59 Friis B, Andersen P, Brenoe E et al. Antibiotic treatment of pneumonia and bronchiolitis. A prospective ran domised study. Arch Dis Child. 1984;59:1038-1045.

60 Kein M. Multicenter trial of cefpodoximeproxetil vs. amoxicillin-davulanatein acutelower respiratory tract infections in childhood. International Study Group. Pediatr Infect Dis J. 1995;14:S19-S22.

61 RodriguezWJ, Guber WC, Goothuis JR et al. Respiratory syncytial virus immunegl obulin treatment of RSV lower respiratory tract infection in previously heathy children. Pediatrics. 1997;100:937-942.

62 RodriguezWJ, Gruber WC, Welliver RC, et al. Respiratory syncytid virus (RSV) immune globulinintravenous the apy for RSV lower respiratory tract infection in infants and young children at high risk for severe RSV infections: Respiratory Syncytial Virus Immune Gobulin Study Goup. Pediatrics. 1997;99:454-461

63 Chipps BE, Sullivan WF, Portnoy JM. Apha-2A interfer on for treatment of bronchiolitis caused by respiratory syncytial virus. Pediatr Infect Dis J. 1993;12:653-658.

64 Hollman G, Shen G Zeng L, ed al. Helium-oxygen improves ainicd AsthmaScores in children with acute bronchiolitis. Oit Care Med. 1998;26:1731-1736.

65 Luchetti M, Casiraghi G, Valsecchi R, Galassini E, Marrao G Porcine derived surfactant treatment of severe bronchiolitis. Acta Anaesthesiol Scand. 1998:42:805-810.

66 Van Bever HP, Desager IN, Pauwds JH Wojciechowski M, Vermere PA. Aerosolized furosemide in wheezy infants: a negativereport. Pediatr Pulmonol. 1995;20:16-20.

67 Nass SZ, Strouse PJ, SoskolneE, et al. Efficacy of recombinant human deoxyribonucleasel in the hospital management of respiratory syncytial virus bronchiolitis. Chest. 2001;120:203-208.

68 Americen Academy of Pediatrics. Respir tory Syncytial Virus. En: Pickering LK, ed. 2000 Red Book: Report of the Committee on Infectious Diseases. 25th ed. Bk Grove Village, II: American Academy of Pediatrics; 2000 484 .

69 Kellner JD, Ohlsson A, Gadomski AM, Wang EE: Eficacy of bronchodilator therapy in bronchiolitis. A meta andysis. Arch Pediat Adolesc Med 1996; 150(11): 1166-1172

Hores G Horwitz Rl. Efficacy of b2-agonists in bronchiolitis: areappraisaland meta-analysis. Pediatrics 1997;100:233-239.

71 Garrison MM, Christakis DA, Harvey E, Oummings P, Davis RL: Systemic corticoster oids in infant bronchiolitis: a meta-analysis. Pediatrics 2000; 105(4): e44.

72 The PREVENT Study Group. Reduction of respiratory syncytia virus hospitalization among premature infants and infants with bronchopulmonary dysplasia using respiratory syncytial virus immune globulin prophylaxis. Pediatrics. 1997;99:93-99.

73 Simoes EA, Sondheimer HM, Top F+N, et al. Respiratory syncytial virus immuneglobulinfor prophylaxis against respiratory syncytial virus disease in inf ants and children with congenital heart disease. The Cardiac Study

74 Groothuis JR, Simoes EA, Levin MJ, et al. Prophylactic administration of respiratory syncytial virus immune globulinto high-riskinfants and young children TheRespiratory Syncytial Virus ImmuneGobulin Study Group. NEngl J Med. 1993;329:1524-1530.

75 The IMpact-RSV Study Group. Palivizumab, a humanized respir atory syncytial virus monodonal antibody, re duces hospitalization from respiratory syncytial virus infection in high-risk infants. Pediatrics. 1998;102:531 537

76 Meissner HC, Groothuis JR, Rodriguez WJ, et al. Satety and phamacokinetics of an intramuscular monoclona antibody (SB209763) against respiratory syncytial virus (RSV) in infants and young children at risk for severe RSV disease. Antimicrob Agents Chemother. 1999;43:1183-1188.

77 Groothuis JR, King SJ, Hogerman DA, Paradiso PR, Simoes EA Safety and immunogenicity of a purified F protein respiratory syncytial virus (PPP-2) vaccine in seropositive children with bronchopulmonary dysplasia J Infect Dis. 1998; 177:467-469.

78 Piedra PA Grace S, Jewell A, et al. Purified fusion protein vaccine protects against lower respiratory tract illness during respiratory syncytial virus season in children with cystic fibrosis. Pediatr Infect Dis J. 1996;15:2331.

79 Pedra PA GraceS, Jewell A, et al. Sequential annual administration of purified fusion protein vaccine against respiratory syncytial virus in children with cystic fibrosis. Pediatr Infect Dis J. 1998;17:217-224.

80 Marchetti A Lau H Magar R, Wang L Deveroelli G Impact of palivizumab on expected costs of respiratory syncytial virus infection in preterm infants: potential for savings. Cin Ther. 1999;21:752-766.

81 Joffe S, Ray GT, Escobar GJ, Black SB, Lieu TA Cost-effectiveness of respiratory syncytial virus prophylaxis among preterm infants. Pediatrics. 1999;104:419-427.

$82 \mathrm{NumaA}$ Outcome of respiratory syncytid virus infection and a cost-benefit analysis of prophylaxis. J Paedia tr Child Health. 2000;36:422-427.

83 Lofland JH, OConnor JP, Chatterton ML, et al. Palivizumab for respiratory syncytial virus prophylaxis in highrisk infants: acost-effectiveness andysis. Oin Ther. 2000;22:1357-1369

84 Schrand LM, Eliott JM, Ross MB, Bell F, Mutnick AH. Cost-benefit analysis of RSV prophylaxis in high-risk infants. Ann Pharmacothe. 2001;35:1186-1193.

85 Fariña D, Rodriguez SP, Bauer G et al. Respiratory syncytial virus prophylaxis: cost-effective analysis in Argentina Pediar Infect Dis J. 2002;21:287-291. 